UW Biostatistics Working Paper Series

$11-1-2007$

\title{
Identifiability and Estimation of Causal Effects in Randomized Trials with Noncompliance and Completely Non-ignorable Missing-Data
}

Hua Chen

Peking University, huachen@u.washington.edu

Zhi Geng

Peking University, zgeng@math.pku.edu.cn

Xiao-Hua Zhou

University of Washington, azhou@u.washington.edu

\section{Suggested Citation}

Chen, Hua; Geng, Zhi; and Zhou, Xiao-Hua , "Identifiability and Estimation of Causal Effects in Randomized Trials with Noncompliance and Completely Non-ignorable Missing-Data" (November 2007). UW Biostatistics Working Paper Series. Working Paper 317.

http://biostats.bepress.com/uwbiostat/paper317

This working paper is hosted by The Berkeley Electronic Press (bepress) and may not be commercially reproduced without the permission of the copyright holder.

Copyright (C) 2011 by the authors 


\section{Introduction}

Two common problems in clinical trials are noncompliance and missing outcome data. Noncompliance occurs when some subjects fail to comply with their assigned treatments; missing data occurs when study investigators cannot collect outcome information on some subjects. Ignoring the noncompliance or missing data may result in biased estimates of causal effects. Moreover, the assumed mechanism of missing-data also has an impact on the estimated causal effects. Many methods have been developed for handling either missing data or noncompliance, but researchers have only recently started to develop methods for handling both missing outcome data and noncompliance in the same study (Frangakis and Rubin, 1999; Zhou and Li, 2006; Yau and Little, 2001; O'Malley and Normand, 2005).

Frangakis and Rubin (1999) proposed a moment estimator for the complier average causal effect (CACE) parameter under the binary compliance status and latent ignorable (LI) missing outcome assumption. The LI assumption means the missing data mechanism has no residual dependence with the outcome, given the observed data and latent compliance class. Under the same LI assumption, Zhou and Li (2006) derived maximum likelihood (ML) estimates as well as moment estimates of the CACE when the compliance status is a discrete variable with three categories and when the outcome variable is binary. O'Malley and Normand (2005) gave the moment and ML estimators of the CACE for a continuous outcome variable.

The above mentioned methods may yield biased estimators of the CACE if the missing data mechanism is a different type of the non-ignorable missing mechanism from latent ignorability. The mechanism of missing outcome $Y$ may depend on missing values of $Y$. For example, some subjects may drop out of a study because of a patient's declining health condition, which is related to $Y$ given the observed data and latent compliance class. As a motivating example, consider a study on the effectiveness of influenza vaccine efficacy 
in reducing morbidity in high-risk adults (McDonald et al., 1992). This study began in 1978 and lasted for three years. There were about two thousand patients enrolled in the study. Physicians were randomly assigned to the treatment group of the control group at the beginning of the study. The physicians assigned to the treatment group would encourage their eligible patients to get a flu shot. But the patients themselves decided whether or not to take flu shots. One of the main outcomes in the study was the flu-related hospitalization. Some patients' outcomes were not observed, and the reason for missing outcomes may depend on the missing values. For example, some subjects were missing their outcomes because they had flu but went to different hospitals than the study hospital, and as a result their outcomes were not recorded. Or, some patients were missing their outcomes because the reason for their hospitalizations was unknown. When the missing data mechanism depends on the outcome, we define this situation as completely non-ignorable (CN). The LI missing data mechanism assumed that subjects dropped out because of subjects' latent compliance statuses.

Analysis of CN missing data is more difficult than analysis of LI data. One major difficulty under the $\mathrm{CN}$ missing data mechanism is the issue of parameter identifiability. Here we say that a parametric model $P_{\theta}$ is identifiable if there is a unique value of the parameter vector $\theta$ that can generate a given observed distribution $F_{\theta}$, that is if $F_{\theta}=F_{\theta^{\prime}}$, then $\theta=\theta^{\prime}$. When the missing-data mechanism is non-ignorable, some of parameters may not be identifiable even if data provide enough degrees of freedom (Little and Rubin, 2004). Several authors have proposed methods for dealing with CN. For example, Brown (1990) developed an estimation method for missing normal outcome variables in longitudinal studies under the CN missing mechanism. Robins and Rotnitzky (2004) discussed the parameter identifiability in randomized trials with non-compliance. Vansteelandt and Goetghebeur (2005) discussed the parameter identifiability in randomized trials with non-compliance and 
missing data. However, no methods are available for dealing with the $\mathrm{CN}$ missing mechanism and noncompliance in the same study.

In this paper we fill the gap by first studying identifiability of the model parameters under the $\mathrm{CN}$ assumption. We show that the parameters are identifiable under two different conditions. The first condition assumes that the missing data mechanism depends only on the missing outcome variable. If this assumption does not hold, the parameters are not identifiable. However, we can show that the parameters are also identifiable if we can find an observed discrete covariate $X$ before the treatment assignment, which associated with $Y$ in each subpopulation of the compliance and treatment level. Then we derive both moment and ML estimators.

The paper is organized as follows. We describe notation and assumptions in Section 2. In Section 3, we give the theoretical results on identifiability of the parameters. In Section 4, we conduct simulation studies to assess the finite-sample properties of the derived estimators and sensitivity of the proposed estimators to the departure from the assumed conditions. Then we illustrate the application of the proposed methods in a real study. We give some concluding remarks in Section 5. The proofs of theorems are presented in Appendix.

\section{Notation and Assumptions}

For the sake of notational simplicity, we suppress the index $i$, which represents the $i$ th patient. Let $Z$ represent the randomized treatment assignment $(1=$ new, $0=$ control $)$ and let $\xi=P(Z=1)$. Let the binary variable $D$ denote which treatment the patient receives (1 =new treatment received, $0=$ control received). Let $D(Z)$ represent which treatment received if the patient's physician is assigned to the treatment $Z$, and $Y$ be a binary outcome variable. $Y=0$ if the patient goes to the hospital due to the flu and $Y=1$ otherwise. $R$ is the binary response indicator of $Y$, that is, $R=1$ if $Y$ is observed and $R=0$ if $Y$ is missing. We define $Y\{Z, D(Z)\}$ or $Y(Z)$ as the potential outcome of the patient if the patient is 
assigned the treatment $Z \cdot R(Z)$ is the potential binary response indicator of a patient if the patient is assigned to the treatment $Z$. In our notation, $Z, R, D$, and $Y$ are observed data of a patient, and $D(Z), Y(Z)$, and $R(Z)$ are potential outcomes of a patient.

Following Imbens and Rubin (1997), we let $U$ be the compliance status of a patient, defined as follows:

$$
U=\left\{\begin{array}{l}
c, \quad \text { if } D(0)=0 \text { and } D(1)=1 \\
n, \quad \text { if } D(0)=0 \text { and } D(1)=0 \\
a, \quad \text { if } D(0)=1 \text { and } D(1)=1 \\
d, \quad \text { if } D(0)=1 \text { and } D(1)=0,
\end{array}\right.
$$

where $c, n, a$ and $d$ represent complier, never-taker, always-taker and defier, respectively. Here $U$ is an unobserved variable, representing compliance behavioral patterns of the patient. Let $\omega_{u}=P(U=u)$. For simplicity, we also denote $\rho_{y z u}=P(R=1 \mid Y=y, Z=z, U=u)$ and $\theta_{y z u}=P(Y=y \mid Z=z, U=u)$. As in Imbens and Rubin (1997) and Frangakis and Rubin (1999), in this paper we consider CACE as the parameter of interest, defined as $C A C E=E\{Y(1)-Y(0) \mid U=c\}$.

Since the joint distribution of the potential outcomes $Y(z), R(z)$ and $U$ conditional on $Z=z$ can be expressed by parameters $\omega_{u}, \rho_{y z u}$ and $\theta_{y z u}$, causal effects are identifiable if we can show these parameters are identifiable. Next, we will give the necessary assumptions to make these parameters identifiable under the $\mathrm{CN}$ missing mechanism.

Assumption 1: Stable unit treatment value assumption (SUTVA) (Rubin, 1978; Angrist et al., 1996; Imbens and Rubin, 1997).

SUTVA implies that potential outcomes do not depend on the treatment status of other individuals.

Assumption 2: Randomization: $Z$ is randomized. 
We can express the CACE as $C A C E=\theta_{11 c}-\theta_{10 c}$ under the randomization assumption.

Assumption 3: Monotonicity: $D_{i}(1) \geq D_{i}(0)$ for all subjects $i$, which implies there are no defiers.

Assumption 4: Exclusion restrictions (Angrist et al., 1996): $P\left\{Y_{i}(1) \mid U_{i}=n\right\}=P\left\{Y_{i}(0) \mid U_{i}=\right.$ $n\}$, and $P\left\{Y_{i}(1) \mid U_{i}=a\right\}=P\left\{Y_{i}(0) \mid U_{i}=a\right\}$.

The exclusion restriction implies $P\left(Y_{i} \mid Z_{i}=1, U_{i}=n\right)=P\left(Y_{i} \mid Z_{i}=0, U_{i}=n\right)$, and $P\left(Y_{i} \mid Z_{i}=\right.$ $\left.1, U_{i}=a\right)=P\left(Y_{i} \mid Z_{i}=0, U_{i}=a\right)$, that is $\theta_{11 n}=\theta_{10 n}$ and $\theta_{11 a}=\theta_{10 a}$. In some studies, such as double blinding, exclusion restrictions are reasonable.

Assumption 5: Compound exclusion restrictions (Frangakis and Rubin, 1999): $P\left\{Y_{i}(1), R_{i}(1) \mid U_{i}=\right.$ $n\}=P\left\{Y_{i}(0), R_{i}(0) \mid U_{i}=n\right\}$, and $P\left\{Y_{i}(1), R_{i}(1) \mid U_{i}=a\right\}=P\left\{Y_{i}(0), R_{i}(0) \mid U_{i}=a\right\}$.

Assumption 5 is stronger than Assumption 4. Besides having the same implications as Assumption 4, Assumption 5 also implies $P\left(R_{i} \mid Z_{i}=1, U_{i}=n\right)=P\left(R_{i} \mid Z_{i}=0, U_{i}=n\right)$ and $P\left(R_{i} \mid Z_{i}=1, U_{i}=a\right)=P\left(R_{i} \mid Z_{i}=0, U_{i}=a\right)$. Assumption 4 instead of Assumption 5 is required in our Theorems 1 and 2 where the missing-data mechanism doesn't depend on latent compliance status variable $U$ (Assumptions 6 and 7). Assumption 5 instead of 4 is required in Theorems 3 where the missing-data mechanism depends on both missing outcomes and $U$ (Assumption 8).

\section{Identifiability and Estimation}

In this section, we discuss additional conditions needed to identify the causal parameters under the CN assumption, and then propose moment and ML estimators of causal effects. Intuition behind how identification of parameters is achieved is related to the idea of instrumental variables. As we know, if there are no missing outcomes, causal parameters are identifiable under the standard assumptions 1 to 4 of an instrumental variable, as shown 
in Angrist, Imbens, and Rubin (1996). When the missing data mechanism depends only on outcomes, under the assumptions 1 to 4 , as well as the assumption $6,(U, Z)$ can be considered as an instrumental variable, and causal parameters are still identifiable. In Section 3.1, we consider a missing-data mechanism model in which the mechanism of missing outcome $Y$ depends only on the outcome $Y$ itself; that is, only the outcome $Y$ has an effect on $R$. Under this assumption we provide a sufficient condition on parameter identifiability in Theorem 1. Without any other assumptions on the missing data mechanism, only this model and latent ignorable model can be identified.

When the missing data mechanism depends on more variables, an additional instrumental variable is required to identify parameters. If the missing-data mechanism depends on not only $Y$ but also the treatment assignment $Z$, we can still identify the parameters in this model when we have one additional covariate $(X)$ that can affect the outcome $Y$ but does not depend on the other variables $D$ and $Z$ in the study. This model is more general than the first missing-data model. Here $(X, U)$ is being used as an instrumental variable for finding the effect of Y on R. In Section 3.2, we present the results under this more general model. In Section 3.3, we extend our identifiability results to an discrete outcome with more than two categories.

\subsection{Identifiability without Covariate}

We consider a CN mechanism which satisfies the following assumption:

Assumption 6: $P\left\{R_{i}(z) \mid Y_{i}(z), D_{i}(z), U=u\right\}=P\left\{R_{i}(z) \mid Y_{i}(z)\right\}$ for $z=0$ and 1 , and $P\left\{R_{i}(1) \mid Y_{i}(1)=y\right\}=P\left\{R_{i}(0) \mid Y_{i}(0)=y\right\}$.

When $Z$ is randomized, Assumption 6 implies $\rho_{y z u}=\rho_{y z^{\prime} u^{\prime}}$ for any $z \neq z^{\prime}$ or $u \neq u^{\prime}$.

Before studying parameter identifiability, we compared Assumption 6 with the LI assumption. The LI assumption requires that potential outcomes and associated potential 
nonresponse indicators are independent within each level of the latent compliance covariate (Frangakis and Rubin, 1999), that is $P\{R(1) \mid U, Y(1)\}=P\{R(1) \mid U\}$ and $P\{R(0) \mid U, Y(0)\}=$ $P\{R(0) \mid U\}$. The LI assumption means patients drop out because of their latent compliance class. Yet Assumption 6 means that patients may drop out because of a worsen disease condition, which is related to the outcome. For example, they may drop out when they feel more terrible after taking the assigned drugs. In our study, $Y$ measures the hospitalization of a subject and the reason for missing $Y$ of a subject may due only to her/his hospitalization status. So whether patients drop out of the trial is determined by their outcomes, not by their inherent and invariable nature. These two assumptions are so different that a wrong assumption will have a serious impact on estimation of $C A C E$ unless $C A C E$ is close to zero. We will see this point in our simulations.

Next theorem shows that the parameters are all identifiable under Assumption 6. For the case of simplicity, we denote $\rho_{y}=\rho_{y z u}$ and $\delta_{y z u}=P(Y=y, R=1 \mid Z=z, U=u)$. Under Assumption 6 , the vector of parameters is $\underline{\boldsymbol{\theta}}=\left(\xi, \omega_{a}, \omega_{n}, \theta_{10 a}, \theta_{11 n}, \theta_{11 c}, \theta_{10 c}, \rho_{0}, \rho_{1}\right)$.

Theorem 1: If $Y$ is not independent of $Z$ given $U$ or if $Y$ is not independent of $U$ given $Z$, then under Assumptions 1-4 and Assumption 6, the vector of parameters, $\underline{\boldsymbol{\theta}}$, is identifiable.

We give a detailed proof of this theorem in Appendix. It is worthwhile to note that if $Y$ is independent of $Z$ given $U$ and is also independent of $U$ given $Z$, we can not identify all the parameters. However, from $\theta_{10 a}=\theta_{11 n}=\theta_{11 c}=\theta_{10 c}$, we can get $C A C E=\theta_{11 c}-\theta_{10 c}=0$, which means the treatment has no causal effect on the outcome.

After we have shown identifiability of $\underline{\boldsymbol{\theta}}$, we can derive the moment and ML estimators of $\underline{\boldsymbol{\theta}}$. Let $N_{y r z d}$ be the observed number of patients with $Y=y, R=r, Z=z, D=d$. The observed data, $N_{y 1 z d}\left(\right.$ for $y, z, d=0,1$ ) and $N_{+0 z d}$ (for $z, d=0,1$ ), can be considered as from a multinomial distribution with the corresponding cell probabilities, $\nu_{y 1 z d}$ and 
$\nu_{+0 z d}$, where $N_{+0 z d}=\sum_{y} N_{y 0 z d}$ denotes an observed frequency with $y$ 's value missing, $\nu_{y 1 z d}=P(Y=y, R=1, Z=z, D=d)$ and $\nu_{+0 z d}=P(R=0, Z=z, D=d)$.

Then the moment estimator of $C A C E$ is $\widehat{C A C E}_{\text {nocova }}=\frac{\widehat{\delta}_{11 c}-\widehat{\delta}_{10 c}}{\widehat{\rho}_{1}}$. And we can obtain that $\widehat{C A C E}_{\text {nocova }}$ has an asymptotically normal distribution using the central limit theorem and the multivariate delta method. Since moment estimates may be the outside of the parameter space in practice (Zhou and Li, 2006), we propose the EM algorithm to find ML estimates in this article. In Theorem 1 the complete-data likelihood function is given as $L_{c}(\theta)=\Pi_{i=1}^{N} P\left(Z_{i}\right) P\left(U_{i}\right) P\left(D_{i} \mid Z_{i}, U_{i}\right) P\left(Y_{i} \mid Z_{i}, U_{i}\right) P\left(R_{i} \mid Y_{i}\right)$. In the $\mathrm{E}$ step, we take the expectation of the complete-data, given the observed data and the previous parameter estimate $\underline{\boldsymbol{\theta}}=\underline{\boldsymbol{\theta}}^{(k)}$, that is $n_{y r z u}^{(k+1)}=E\left\{n_{\text {yrzu }} \mid\right.$ observed-data, $\left.\underline{\boldsymbol{\theta}}^{(k)}\right\}$. In the M step, we can get the ML estimates $\underline{\boldsymbol{\theta}}^{(k+1)}$ from the $n_{y r z u}^{(k+1)}$.

\subsection{Identifiability with a Covariate}

In some clinical trials there are good reasons to believe that the missing data mechanism is also affected by the treatment assignment not just the outcome, because the occurrence of side effects differs between treatment arms. In some clinical trials, a direct effect of the treatment assignment on the missing data mechanism is essentially implied by the study design. For example, when patients in the treatment group experience severe side effects, they are removed from further study. Therefore, the response indicator of the outcome, $R$, depends not only on the outcome $Y$ itself but also other variables. The parameter vector $\underline{\boldsymbol{\theta}}$ is not identifiable under only Assumptions 3, 4, and 6 without further assumptions. In this case, we can introduce an additional covariate $X$ which is observed before the assigned treatment, and thus $Z$ is independent of $(X, U)$. Suppose that $X$ associated with $Y$ in each subpopulation of $U=u$ and $Z=z$ (that is, $P(y \mid x, z, u) \neq P(y \mid z, u)$ for some $x$ and for all $u$ and $z$ ) such that the parameter vector $\underline{\boldsymbol{\theta}}$ becomes to be identifiable. For example, in some clinical trials it may be reasonable to assume that the age of patients is associated with $Y$ in each subpopulation. 
Here we suppose that $x$ is discrete. Then the following theorem shows that parameters are identifiable. Let $\alpha_{x u}=P(X=x, U=u), \rho_{y z u x}=P(R=1 \mid Y=y, Z=z, U=u, X=x)$ and $\theta_{y z u x}=P(Y=y \mid Z=z, U=u, X=x)$. To emphasize the dependence of the causal effect parameter on covariates, we write $C A C E$ as $C A C E_{\text {cova }}$, which is defined as follows: $C A C E_{\text {cova }}=\sum_{x} E\{Y(1)-Y(0) \mid U=c, X=x\} P(X=x \mid U=c)=\sum_{x}\left(\theta_{11 c x}-\theta_{10 c x}\right) \alpha_{x c} / \omega_{c}$.

With availability of this covariate $X$, we can replace Assumption 6 by the following assumption.

Assumption 7: For $z=0$ and 1,

$$
P\left\{R_{i}(z) \mid Y_{i}(z), D_{i}(z), U=u, X=x\right\}=P\left\{R_{i}(z) \mid Y_{i}(z)\right\}
$$

Assumption 7 means that the missing data mechanism depends on both the outcome $Y$ and the assigned treatment $Z$, which is weaker than Assumption 6. To identify parameters under Assumption 7, we introduce an observed covariate $X$ as an additional instrumental variable in Theorem 2.

When the treatment assignment $Z$ is randomized, we have from (1) that $\rho_{y z u x}=\rho_{y z u^{\prime} x^{\prime}}$ for any $u \neq u^{\prime}$ or $x \neq x^{\prime}$, and thus we can simply denote $\rho_{y z u x}$ as $\rho_{y z}$. The vector of parameters, $\underline{\boldsymbol{\theta}}$, is denoted as $\underline{\boldsymbol{\theta}}=\left(\xi, \omega_{a}, \omega_{n}, \alpha_{x a}, \alpha_{x n}, \alpha_{x c}, \theta_{10 a x}, \theta_{11 n x}, \theta_{11 c x}, \theta_{10 c x}, \rho_{00}, \rho_{01}, \rho_{10}, \rho_{11}\right)$.

Theorem 2: Suppose that $X$ is an observed discrete covariate that depends on $Y$ in each subpopulation of $U=u$ and $Z=z$. Then under Assumptions 1-4 and Assumption 7, the vector of parameters, $\underline{\boldsymbol{\theta}}$, is identifiable.

We give a proof of Theorem 2 in the appendix. Under the model in Theorem 2, we can obtain the estimate of $C A C E$ as $\widehat{C A C E}_{\text {cova } 1}=\sum_{x}\left(\widehat{\delta}_{11 c x} / \widehat{\rho}_{11}-\widehat{\delta}_{10 c x} / \widehat{\rho}_{10}\right) \widehat{\alpha}_{x c} / \widehat{\omega}_{c}$.

Note that in Theorems 1 and 2 we only make the exclusion restriction assumption, which is weaker than the compound exclusion restriction assumption made in Frangakis and Rubin (1999). If we also make the stronger compound exclusion assumption, we can further relax 
Assumption 7 to allow the missing-data mechanism to depend on both missing outcomes and latent compliance status variable.

Assumption 8: For $z=0$ and 1 ,

$$
P\left\{R_{i}(z) \mid Y_{i}(z), D_{i}(z), U=u, X=x\right\}=P\left\{R_{i}(z) \mid Y_{i}(z), U=u\right\}
$$

This assumption assumes that the missing data mechanism depends on $Y, Z$ and $U$. When the treatment assignment $Z$ is randomized, using (2) we obtain that $\rho_{y z u x}=\rho_{y z u x^{\prime}}$ for any $x \neq x^{\prime}$, and thus we can simply denote $\rho_{y z u x}$ as $\rho_{y z u}$. Since the compound exclusion assumption (Assumption 5) holds, we have that $\rho_{y 0 n}=\rho_{y 1 n}$ and $\rho_{y 0 a}=\rho_{y 1 a}$. Hence, the vector of parameters, $\underline{\boldsymbol{\theta}}$, is $\underline{\boldsymbol{\theta}}=\left(\xi, \omega_{a}, \omega_{n}, \alpha_{x a}, \alpha_{x n}, \alpha_{x c}, \theta_{10 a x}, \theta_{11 n x}, \theta_{11 c x}, \theta_{10 c x}, \rho_{11 n}, \rho_{01 n}, \rho_{00 a}, \rho_{10 a}, \rho_{11 c}, \rho_{01 c}, \rho_{00 c}, \rho_{10 c}\right)$.

TheOREm 3: Suppose that $X$ is an observed discrete covariate that depends on $Y$ in each subpopulation of $U=u$ and $Z=z$. Then under Assumptions 1-3, 5, and 8, the parameters in $\underline{\boldsymbol{\theta}}$ are identifiable.

The difference between the models in Theorems 2 and 3 is in their missing data mechanisms. For the model in Theorem 3, $R$ depends on $Y(Z), Z$ and $U$, while $R$ depends only on $Y(Z)$ and $Z$ in the model of Theorem 2. For the model of Theorem 3, we can obtain the estimate of $C A C E$ as $\widehat{C A C E}_{\text {cova2 }}=\sum_{x}\left(\widehat{\delta}_{11 c x} / \widehat{\rho}_{11 c}-\widehat{\delta}_{10 c x} / \widehat{\rho}_{10 c}\right) \widehat{\alpha}_{x c} / \widehat{\omega}_{c}$.

\subsection{Extension to multi-level outcomes}

In this subsection we generalize Theorems 1,2 , and 3 to a multi-level outcome. Let $Y$ be a K-level discrete variable, where $Y=0, \ldots, K-1$, and the covariate $X$ be a $J$-valued variable, i.e., $X \in\{0,1, \ldots, J-1\}$. Since the proofs of corollaries are similar to theorems, we omit the proofs for the sake of simplicity.

COROllary 1: If $Y$ has fewer levels than 5, that is $K<5$, and the rank of the $4 \times K$ 
matrix,

$$
\left(\begin{array}{llll}
\delta_{01 n} & \delta_{11 n} & \ldots & \delta_{K-1,1 n} \\
\delta_{00 a} & \delta_{10 a} & \ldots & \delta_{K-1,0 a} \\
\delta_{01 c} & \delta_{11 c} & \ldots & \delta_{K-1,1 c} \\
\delta_{00 c} & \delta_{10 c} & \ldots & \delta_{K-1,0 c}
\end{array}\right)
$$

is equal to $K$, then the result of Theorem 1 holds.

Note if $K>4$, the model of Theorem 1 cannot be identified without additional assumptions, because the degree of freedom in the observed data is $4 K+3$, which is smaller than the number of parameters $5 K-1$.

Corollary 2: Let us define the following $J \times K$ matrices:

$$
\underline{\Delta}_{z u}^{\text {Multi }}=\left(\begin{array}{cccc}
\delta_{0 z u 0} & \delta_{1 z u 0} & \ldots & \delta_{K-1, z u 0} \\
\delta_{0 z u 1} & \delta_{1 z u 1} & \ldots & \delta_{K-1, z u 1} \\
\vdots & \vdots & \ldots & \vdots \\
\delta_{0 z u, J-1} & \delta_{1 z u, J-1} & \ldots & \delta_{K-1, z u, J-1}
\end{array}\right)
$$

where $u=n, a, c$ and $z=0,1$.

(1) When $J \geq K$, if the ranks of the two $J \times K$ matrices, $\underline{\Delta}_{1 n}^{\text {Multi }}$ and $\underline{\Delta}_{0 a}^{\text {Multi }}$, are equal to $K$, then the result of Theorem 2 holds.

(2) When $J \geq K$, if the ranks of the four $J \times K$ matrices, $\underline{\Delta}_{1 n}^{\text {Multi }}, \underline{\Delta}_{1 c}^{\text {Multi }}, \underline{\Delta}_{0 a}^{\text {Multi }}$ and $\underline{\Delta}_{0 c}^{\text {Multi }}$, are all equal to $K$, then the result of Theorem 3 holds.

\section{Simulation Studies and Application}

In our simulation studies, we first assessed the relative performance of the moment and ML estimators in finite-sample sizes when the assumptions were correct. We then assessed the sensitivity of the derived moment and ML estimators when some of the assumptions were violated. 
In the first simulation study, we generated 1000 samples. Each of which had a sample size of $N=500$ under the model with a covariate as specified in Theorems 2 . We computed moment and ML estimates of parameters for every sample, their means, standard deviations, and actual coverage percentages of $95 \%$ confidence intervals. The result is reported in Table 1. We used the bootstrap to estimate the standard deviation. Since the moment and ML estimates are all asymptotically normal distributions, we also computed confidence intervals. We also generated data under the missing-data models, given in Theorems 1 and 3 . Since the results were similar to that in Theorem 2 , we only reported the results on the missing-data model with a covariate in Theorem 2 for the sake of simplicity. From Table 1, we see that except for $\xi, \omega_{n}$ and $\omega_{a}$, the ML estimates perform better than the moments estimators. In addition, for half of the samples the moment estimates are not proper (meaning that at least one of the estimates for the sample is outside of the corresponding parameter's range). Hence we would recommend the ML estimates over the moment estimates.

[Table 1 about here.]

Next we conducted a sensitivity analysis of the proposed estimators between the LI assumption and CN assumption. We assumed that the true model satisfied the CN assumption described in Theorem 1, but we estimated the $C A C E$ under the wrong LI assumption. Thus the true $C A C E_{\text {true }}$ is $\theta_{11 c}-\theta_{10 c}$ and the estimated $C A C E_{\text {estimated }}$ is $\frac{\theta_{11 c} \rho_{1}}{\theta_{11 c} \rho_{1}+\left(1-\theta_{11 c}\right) \rho_{0}}-$ $\frac{\theta_{10 c} \rho_{1}}{\theta_{10 c} \rho_{1}+\left(1-\theta_{10 c}\right) \rho_{0}}$. Let bias $=\left|C A C E_{\text {estimated }}-C A C E_{\text {true }}\right|$. We maximized bias over all values from 0.0 to 1.0 by step 0.01 of $\theta_{11 c}, \theta_{10 c}, \rho_{1}$ and $\rho_{0}$. The result was reported in Figure 1 . Each curve in Figure 1 represents a fixed $C A C E$ value, which was set to be 0.05, 0.1, 0.15, 0.2, and 0.25, respectively. For each of the five true $C A C E_{\text {true }}$ values, we plotted a curve to represent the relationship between the maximum bias of $C A C E$ estimates and a real parameter $|P(R=1 \mid Y=1)-P(R=1 \mid Y=0)|$ in Figure 1. Here, $|P(R=1 \mid Y=1)-P(R=1 \mid Y=0)|$ can be interpreted as a measure for the departure of the assumed LI model from the true CN 
model. The larger $|P(R=1 \mid Y=1)-P(R=1 \mid Y=0)|$ is, the further away the assumed LI model is from the true CN model. From Figure 1, we see that the further away the assumed LI model is from the true $\mathrm{CN}$ mode, the bigger the bias of the CACE estimates obtained under the wrong $L I$ model is. From Figure 1, we can also see that the bias of estimated $\mathrm{CACE}$ depends on the value of the true CACE. In general, the larger the true CACE is, the bigger the bias of the estimated $\mathrm{CACE}$ is. As the true $C A C E$ decreases, the bias of the estimated $C A C E$ also decreases, and as the CACE value tends to zero, the difference between the estimates of the CACE under the CN and LI models also tends to zero.

[Figure 1 about here.]

[Figure 2 about here.]

In Figure 2, we assumed that the true missing-data mechanism model was the LI model, but we used a wrong $\mathrm{CN}$ model to estimate the CACE. Thus the true $C A C E_{\text {true }}$ is $\theta_{11 c}-\theta_{10 c}$ and the estimated $C A C E_{\text {estimated }}$ is $\frac{\left(1-\theta_{11 n}\right) \gamma_{1 n}-\left(1-\theta_{10 a}\right) \gamma_{0 a}}{\gamma_{1 n} \gamma_{0 a}\left(\theta_{10 a}-\theta_{11 n}\right)}\left(\theta_{11 c} \gamma_{1 c}-\theta_{10 c} \gamma_{0 c}\right)$, where $\gamma_{z u}=P(R=$ $1 \mid Z=z, U=u)$. The bias is still denoted as bias $=\left|C A C E_{\text {estimated }}-C A C E_{\text {true }}\right|$. We maximized bias over all values from 0.0 to 1.0 by step 0.01 of $\theta_{11 c}, \theta_{10 c}, \theta_{11 n}, \theta_{10 a}, \rho_{1 n}, \rho_{0 a}$, $\rho_{1 c}$ and $\rho_{0 c}$. We also found that the maximum bias of the estimated $C A C E$ was increasing as the value of the true $C A C E$ was increasing. Thus, the estimate of $C A C E$ is sensitive to the model of the missing data mechanism. The estimate of the $C A C E$ obtained from the LI model is biased if the true missing-data mechanism is a $C N$ model, and vice versa. On the other hand, if the value of $C A C E$ tends to zero, the maximum bias tends to zero regardless whether the true missing-data mechanism model is the $C N$ model or the LI model. Here the estimate of $C A C E$ under the assumption LI is given by Zhou and Li (2006), and the estimate of $C A C E$ under the assumption $\mathrm{CN}$ is given by Theorem 1 in this paper.

We also compared our method with the method in which subjects with missing data were discarded. We generated 1000 samples with sample size $N=3000$ under the model 
of Theorem 1. In Table 2, we reported means and standard errors of estimates of $C A C E$, derived using our method and the method by discarding subjects with missing data. We fix $\rho_{0}=0.1$ and change $\rho_{1}$ from 0.2 to 0.9 and other parameters are fixed as $\xi=0.5, \omega_{n}=0.2$, $\omega_{a}=0.3, \theta_{10 a}=0.6, \theta_{11 n}=0.3, \theta_{11 c}=0.8$ and $\theta_{10 c}=0.2$. From Table 2, we can see that the bias of $C A C E$ estimates obtained by discarding subjects with missing data increases as $\left|\rho_{1}-\rho_{0}\right|$ is increasing; whereas the estimates obtained by our method are very close to the true $C A C E$ regardless of the value of $\left|\rho_{1}-\rho_{0}\right|$.

[Table 2 about here.]

[Table 3 about here.]

Now we apply our method to flu shot data, Zhou and Li (2006). We assume the CN missingdata mechanism satisfying Assumption 6. The observed data are $N_{1100}=49, N_{0100}=573$, $N_{1101}=16, N_{0101}=143, N_{1110}=47, N_{0110}=499, N_{1111}=20, N_{0111}=256, N_{+000}=492$, $N_{+001}=17, N_{+010}=497, N_{+011}=9$. We report the results in Table 3. Since the moment estimates are not proper, we only summarize the ML method. From the table, $\widehat{\rho}_{0}=1$ and the variance is equal to zero. This result means that all patients who were in hospital must be observed.

The estimated CACE and its $95 \%$ confidence interval are -0.1393 and $(-0.4808,0.2022)$ respectively. For a comparison purpose, we listed the estimated CACE and its $95 \%$ confidence interval from Zhou and Li (2006) under the LN assumption. Under latent ignorability, the estimated CACE is -0.009 , and its associated 95\% confidence interval of CACE is ($0.211,0.229)$. Both methods reached the same conclusion that influenza vaccination is not associated with reduced risk of hospitalization for respiratory illness.

There are several limitations to the results of this application. First, we ignore clustering effect in the data that may lead to violation of the SUTVA assumption. Second, since the study is not double blind, the exclusion restrictions assumption may be questionable, 
particularly among the always-takers, who are probably at high risk for flu and then may receive other medical actions beside flu shots given by their physicians when their physicians received a reminder about flu shots.

\section{Conclusion}

In this paper we discussed the problem of non-compliance and non-ignorable missing outcome mechanism. One major problem dealing with a non-ignorable missing data is the issue of parameter identifiability. We gave sufficient conditions for identifying causal effect parameters under the $\mathrm{CN}$ missing-data mechanism, which is one type of non-ignorable missing and is different from the existing LI assumption. Under the CN missing-data mechanism, we give a theorem on parameter identification when the missing data mechanism depends only on outcomes. With availability of a certain type of covariates, we can relax the missing data mechanism assumption - Assumption 6 - to allow the missing-data mechanism to depend on not only the missing outcome variable but also the treatment assignment $Z$ and the latent compliance status variable $U$. From the simulation results, the estimate of CACE is sensitive to the missing data mechanism assumption. So we should pay attention to choose the missing data mechanism in practice. It is still an open problem that how to test a nonignorable missing data mechanism. However, we can obtain from the simulations that the CACE estimates is not sensitive to the missing data mechanism assumption as the true CACE value tends to zero. Some assumptions in our theorems are (partly) testable from the observed data, such as $X$ is not associated with $(D, Z)$.

\section{Acknowledgements}

We would like to thank referees for their valuable comments and suggestions that greatly improved the presentation and structure of this paper. This work was supported in part by 
NIH/NHLBI grant R01HL62567, NSFC, NBRP 2003CB715900 and IAD-06-088. It does not necessarily represent the views of VA HSR\&D Service.

\section{References}

Angrist, J. D., Imbens, G.W. and Rubin, D. B. (1996). Identification of causal effects using instrumental variables (with Discussion). Journal of the American Statistical Association 91, 444-472.

Brown, C. H. (1990). Protecting Against Nonrandomly Missing Data in Longitudinal Studies. Biometrics 46, 143-155.

Frangakis, C. E. and Rubin, D. B. (1999). Addressing complications of intention-to-treat analysis in the combined presence of all-or-none treatment-noncompliance and subsequent missing outcomes. Biometrika 86, 365-379.

Imbenss, G. W. and Rubin, D. B. (1997). Bayesian inference for causal efects in randomized experiments with noncompliance. The Annals of Statistics 25, 305-327.

Little, R. J. A. and Rubin, D. B. (2004). Statistical Analysis with Missing Data Second Edition. John Wiley \& Sons; New York, NY.

McDonald, C.J., Hui, S. L., and Tierney, W. M. (1992). Effects of computer reminders for influenza vaccination on morbidity during influenza epidemics. M. D. Computing 9, 304312.

O'Malley, A. J. and Normand, S-L. T. (2005). Likelihood Methods for Treatment Noncompliance and Subsequent Nonresponse in randomized Trials. Biometrics 61, 325-334.

Robins, J. and Rotnitzky, A. (2004). Estimation of treatment effects in randomized trials with non-compliance and a dichotomous outcome using structural mean models. Biometrika 91, 763-783.

Vansteelandt, S. and Goetghebeur, E. (2005). Sense and sensitivity when correcting for observed exposures in randomised clinical trials. Statistics in Medicine 24, 191-210. 
Yau, L. H. Y. and Little, R. J. (2001). Inference for the complier-average causal effect from longitudinal data subject to noncompliance and missing data, with application to a job training assessment for the unemployed. Journal of the American Statistical Association 96, 1232-1244.

Zhou, X.H. and Li,S.M. (2006). ITT Analysis of Randomized Encouragement Design Studies with Missing-Data. Statistics in Medicine 25, 2737-2761.

\section{Appendix}

\section{Proof of Theorem 1:}

Identifiability of $\xi, \omega_{a}$ and $\omega_{n}$ is immediate from randomization of $Z$ and the monotonicity assumption, that is, $\xi=P(Z=1), \omega_{a}=P(U=a)=P(D=1 \mid Z=0)$ and $\omega_{n}=P(U=$ $n)=P(D=0 \mid Z=1)$. We next show that $\delta_{y z u}$ are the functions of the distributions of observed variables. Under the Assumption 3, we obtain that $\delta_{y 1 n}=P(Y=y, R=1 \mid Z=$ $1, U=n)=\frac{P(Y=y, R=1, Z=1, D=0)}{P(Z=1, D=0)}$ and that $\delta_{y 0 a}=\frac{P(Y=y, R=1, Z=0, D=1)}{P(Z=0, D=1)}$.

For $\delta_{y 1 c}$, we have $\delta_{y 1 c}=\frac{P(Y=y, R=1, Z=1, U=c)}{P(Z=1, U=c)}=\frac{P(Y=y, R=1, Z=1, D=1)-P(Y=y, R=1, D=1, Z=1, U=a)}{P(Z=1, D=1)-P(D=1, Z=1, U=a)}$. Under the monotonicity and randomization assumptions, $P(D=1, Z=1, U=a)$ in the denominator can be rewritten as $P(Z=1) P(D=1, U=a \mid Z=1)=P(Z=1) P(U=a \mid Z=$ $1)=P(Z=1) P(U=a \mid Z=0)=P(Z=1) P(D=1, U=a \mid Z=0)=P(Z=1) P(D=$ $1 \mid Z=0)$. On the other hand, from the numerator we have that $P(Y=y, R=1, D=1, Z=$ $1, U=a)=P(R=1 \mid Y=y, D=1, Z=1, U=a) P(Y=y \mid D=1, Z=1, U=a) P(D=$ $1, Z=1, U=a)$, where $P(R=1 \mid Y=y, D=1, Z=1, U=a)=P(R=1 \mid Y=y)=P(R=$ $1 \mid Y=y, D=1, Z=0, U=a)$ because of Assumption $6, P(Y=y \mid D=1, Z=1, U=a)=$ $P(Y=y \mid D=1, Z=0, U=a)$ due to the exclusion restriction and $P(D=1, Z=1, U=$ a) $=P(D=1, U=a \mid Z=0) P(Z=1)$ by the forward proof. So $P(Y=y, R=1, D=$ $1, Z=1, U=a)=P(R=1 \mid Y=y, D=1, Z=0, U=a) P(Y=y \mid D=1, Z=0, U=$ 
a) $P(D=1, U=a \mid Z=0) P(Z=1)=P(Y=y, R=1, D=1, U=a \mid Z=0) P(Z=1)$.

Hence, we obtain that $\delta_{y 1 c}=\frac{P(Y=y, R=1, Z=1, D=1)-P(Y=y, R=1, D=1 \mid Z=0) P(Z=1)}{P(Z=1, D=1)-P(D=1 \mid Z=0) P(Z=1)}$.

Similarly, we can show that $\delta_{y 0 c}=\frac{P(Y=y, R=1, Z=0, D=0)-P(Y=y, R=1, D=0 \mid Z=1) P(Z=0)}{P(Z=0, D=0)-P(D=0 \mid Z=1) P(Z=0)}$. Hence, we have shown that $\delta_{y z u}$ 's are identifiable.

Next we will show that $\rho_{y}$ 's are identifiable. Let us define the matrix $\underline{\Delta}^{1}$ as follows:

$$
\underline{\boldsymbol{\Delta}}^{1}=\left(\begin{array}{llll}
\delta_{01 n} & \delta_{00 a} & \delta_{01 c} & \delta_{00 c} \\
\delta_{11 n} & \delta_{10 a} & \delta_{11 c} & \delta_{10 c}
\end{array}\right)^{T} .
$$

Because $\theta_{0 z u}+\theta_{1 z u}=1$ and from $\delta_{y z u}=\rho_{y} \theta_{y z u}$, we obtain the following equations:

$$
\underline{\Delta}^{1}\left(\begin{array}{c}
1 / \rho_{0} \\
1 / \rho_{1}
\end{array}\right)=\left(\begin{array}{llll}
1 & 1 & 1 & 1
\end{array}\right)^{T} .
$$

Below we show that $\underline{\Delta}^{1}$ has rank 2 . Suppose that $\underline{\Delta}^{1}$ does not have full column rank. Then we have $\frac{\delta_{01 n}}{\delta_{11 n}}=\frac{\delta_{00 a}}{\delta_{10 a}}=\frac{\delta_{01 c}}{\delta_{11 c}}=\frac{\delta_{00 c}}{\delta_{10 c}}$, which implies $\frac{\theta_{01 n}}{\theta_{11 n}}=\frac{\theta_{00 a}}{\theta_{10 a}}=\frac{\theta_{01 c}}{\theta_{11 c}}=\frac{\theta_{00 c}}{\theta_{10 c}}$ since $\delta_{y z u}=\rho_{y} \theta_{y z u}$. Thus we obtain that $\theta_{10 a}=\theta_{11 n}=\theta_{11 c}=\theta_{10 c}$, which implies that $Y$ is independent of $Z$ given $U$ and is also independent of $U$ given $Z$. This contradicts the condition of Theorem 1. Therefore, we have shown that $\rho_{y}$ 's are identifiable. Finally, the parameters $\theta_{y z u}$ can be identified from equations: $\theta_{10 n}=\theta_{11 n}=\delta_{11 n} / \rho_{1}, \theta_{11 a}=\theta_{10 a}=\delta_{10 a} / \rho_{1}, \theta_{11 c}=\delta_{11 c} / \rho_{1}$ and $\theta_{10 c}=\delta_{10 c} / \rho_{1}$

\section{Proof of Theorem 2:}

The joint distribution can be factorized as $P(Z, U, D, X, Y, R)=P(R \mid Z, U, D, X, Y) P(Y \mid Z, U, D, X)$ $P(D \mid Z, U, X) P(U, X \mid Z) P(Z)$. Since $Z$ is randomized, $P(U, X \mid Z)=P(U, X)$. Because $D$ is determined by $(Z, U)$, we obtain that $P(D \mid Z, U, X)=P(D \mid Z, U)$ and $P(Y \mid Z, U, D, X)=$ $P(Y \mid Z, U, X)$. From the randomization assumption and Assumption 7, we obtain that $P(R \mid Z, U, D, X, Y)=P(R \mid Y, Z)$. So we can rewrite the joint distribution as

$$
P(Z, U, D, X, Y, R)=P(Z) P(U, X) P(D \mid Z, U) P(Y \mid Z, U, X) P(R \mid Y, Z) .
$$


To identify $P(U, X)$, we first note that from independence of $Z$ and $(U, X)$, the definition of $U$, and Assumption 3, we obtain that $P(U=a \mid X=x)=P(U=a \mid Z=0, X=x)=P(D=$ $1 \mid Z=0, X=x), P(U=n \mid X=x)=P(U=n \mid Z=1, X=x)=P(D=0 \mid Z=1, X=x)$ and then $P(U=c \mid X=x)=1-\{P(U=n \mid X=x)+P(U=a \mid X=x)\}$. Since $P(X)$ is identifiable, $P(U, X)$ is identifiable; since $Z$ and $U$ determine $D, P(D \mid Z=z, U=u)$ is known for all $z$ and $u$.

Below we show that $\rho_{01}, \rho_{11}, \rho_{00}, \rho_{10}, \theta_{11 n x}, \theta_{10 a x}, \theta_{11 c x}$ and $\theta_{10 c x}$ are identifiable conditionally on $X=x$. Let us define the following matrices:

$$
\underline{\Delta}_{z u}^{2}=\left(\begin{array}{cc}
\delta_{0 z u x} & \delta_{1 z u x} \\
\delta_{0 z u x^{\prime}} & \delta_{1 z u x^{\prime}}
\end{array}\right)
$$

Since

$$
\delta_{y z u x}=P(R=1 \mid Y=y, Z=z) P(Y=y \mid Z=z, U=u, X=x)=\rho_{y z} \theta_{y z u x},
$$

using the same idea as in the proof of Theorem 1, we obtain the following equations:

$$
\underline{\Delta}_{1 n}^{2}\left(\begin{array}{c}
1 / \rho_{01} \\
1 / \rho_{11}
\end{array}\right)=\left(\begin{array}{c}
1 \\
1
\end{array}\right)
$$

and

$$
\underline{\Delta}_{0 a}^{2}\left(\begin{array}{c}
1 / \rho_{00} \\
1 / \rho_{10}
\end{array}\right)=\left(\begin{array}{c}
1 \\
1
\end{array}\right) .
$$

Under Assumption 3, the elements in the matrices $\underline{\Delta}_{1 n}^{2}$ and $\underline{\Delta}_{0 a}^{2}$ can be expressed by the distributions of observed variables, respectively, as follows:

$$
\delta_{y 1 n x}=\frac{P(Y=y, R=1, Z=1, D=0, X=x)}{P(Z=1, D=0, X=x)}
$$

and

$$
\delta_{y 0 a x}=\frac{P(Y=y, R=1, Z=0, D=1, X=x)}{P(Z=0, D=1, X=x)} .
$$

Suppose that $\underline{\Delta}_{1 n}^{2}$ in (A.3) is not full rank for all $x \neq x^{\prime}$. Then it is immediate that $Y$ is independent of $X$ given $U=n$ and $Z=z$, which contradicts the assumptions in Theorem 2 . 
Thus there exists at least one pair of $x$ and $x^{\prime}$ so that the matrix $\underline{\Delta}_{1 n}^{2}$ is full rank, and then $\rho_{01}$ and $\rho_{11}$ can be solved from (A.3). Similarly, we can show that $\rho_{00}$ and $\rho_{10}$ are identifiable from (A.4).

From (A.2), (A.5) and (A.6), we can identify $\theta_{y 1 n x}$ and $\theta_{y 0 a x}$. Similarly we can identify $\theta_{y 1 c x}$ and $\theta_{y 0 c x}$ from the equations $\theta_{y 1 c x}=\frac{\delta_{y 1 c x}}{\rho_{y 1}}=\frac{P(y, R=1, Z=1, D=1, x) /\left(\xi \rho_{y 1}\right)-P(y, R=1, Z=0, D=1, x) /\left\{(1-\xi) \rho_{y 0}\right\}}{P(Z=1, D=1, x) / \xi-P(D=1, Z=0, x) /(1-\xi)}$ and $\theta_{y 0 c x}=\frac{P(y, R=1, Z=0, D=0, x) /\left\{(1-\xi) \rho_{y 0}\right\}-P(y, R=1, Z=1, D=0, x) /\left(\xi \rho_{y 1}\right)}{P(Z=0, D=0, x) /(1-\xi)-P(D=0, Z=1, x) / \xi}$.

Proof of Theorem 3:

Similar to the proof of Theorem 2, we have $P(Z, U, D, X, Y, R)=P(Z) P(U, X) P(D \mid Z, U)$ $P(Y \mid Z, U, X) P(R \mid Y, Z, U)$. Hence, we can identify $\delta_{y 1 n x}$ and $\delta_{y 0 a x}$ from (A.5) and (A.6) respectively. We can also identify $\delta_{y 1 c x}$ and $\delta_{y 0 c x}$ by $\delta_{y 1 c x}=\frac{P(y, R=1, Z=1, D=1, x)-P(y, R=1, D=1, Z=0, x) \xi /(1-\xi)}{P(Z=1, D=1, x)-P(D=1, Z=0, x) P(Z=1) / P(Z=0)}$ and $\delta_{y 0 c x}=\frac{P(y, R=1, Z=0, D=0, x)-P(y, R=1, D=0, Z=1, x)(1-\xi) / \xi}{P(Z=0, D=0, x)-P(D=0, Z=1, x) P(Z=0) / P(Z=1)}$.

Next we show that we can identify $\rho_{y z u}$. We first put $\delta_{y z u x}$ 's into the following matrices:

$$
\underline{\Delta}_{z u}^{3}=\left(\begin{array}{ll}
\delta_{0 z u x} & \delta_{1 z u x} \\
\delta_{0 z u x^{\prime}} & \delta_{1 z u x^{\prime}}
\end{array}\right),
$$

for $(z, u)=(1, n),(1, c),(0, a)$ and $(0, c)$. Because $R$ is independent of $X$ given $(Y, Z, U)$, we obtain that $\delta_{y z u x}=P(R=1 \mid Y=y, Z=z, U=u) P(Y=y \mid Z=z, U=u, X=x)=$ $\rho_{y z u} \theta_{y z u x}$. Hence, we can obtain the following equations:

$$
\underline{\Delta}_{z u}^{3}\left(\begin{array}{c}
1 / \rho_{0 z u} \\
1 / \rho_{1 z u}
\end{array}\right)=\left(\begin{array}{c}
1 \\
1
\end{array}\right) .
$$

Using the same argument as in the proof of Theorem 2, we can show that all four $\underline{\Delta}_{z u}^{3}$ matrices have full ranks under the assumptions in Theorem 3. Therefore, we can identify $\rho_{y z u}$ 's. Finally, because $\delta_{y z u x}=P(R=1 \mid Y=y, Z=z, U=u) P(Y=y \mid Z=z, U=u, X=x)=\rho_{y z u} \theta_{y z u x}$, we can identify $\theta_{10 a x}, \theta_{11 n x}, \theta_{11 c x}$ and $\theta_{10 c x}$ respectively. 
Figure 1. Real model: CN; Supposed model: LI.

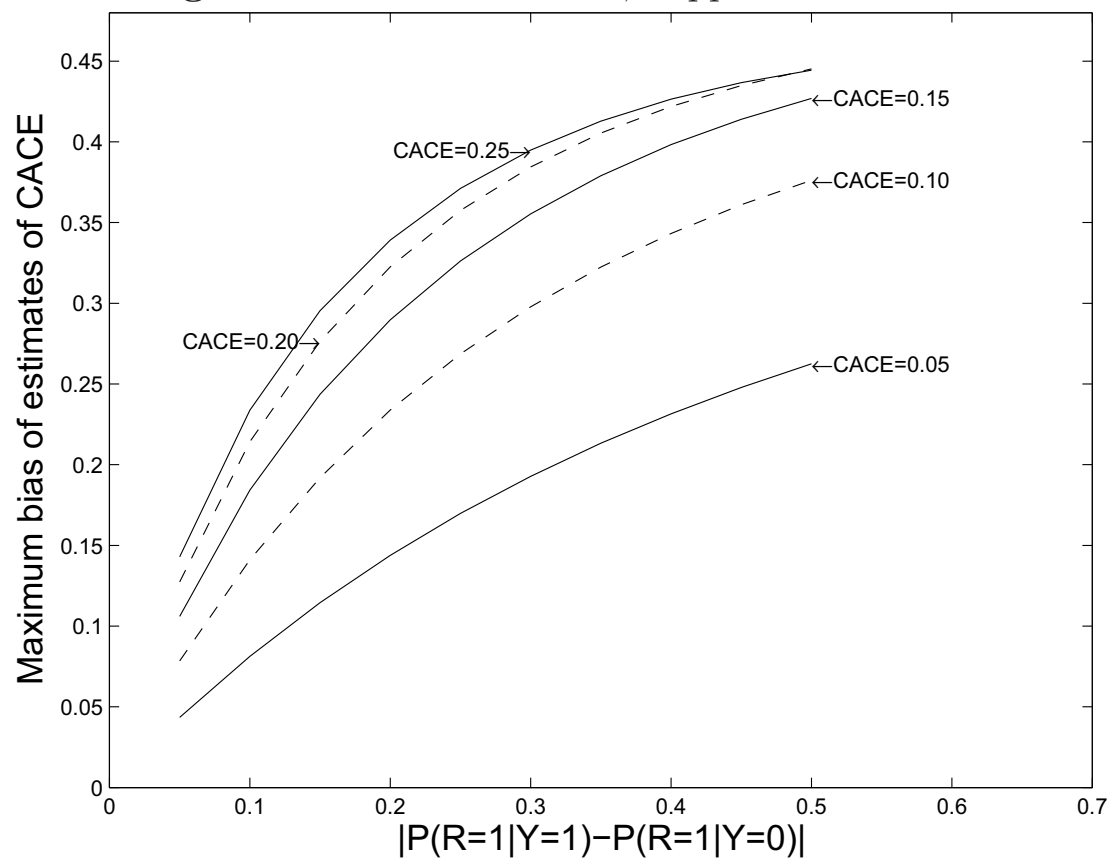


Figure 2. Real model: LI; Supposed model: CN.

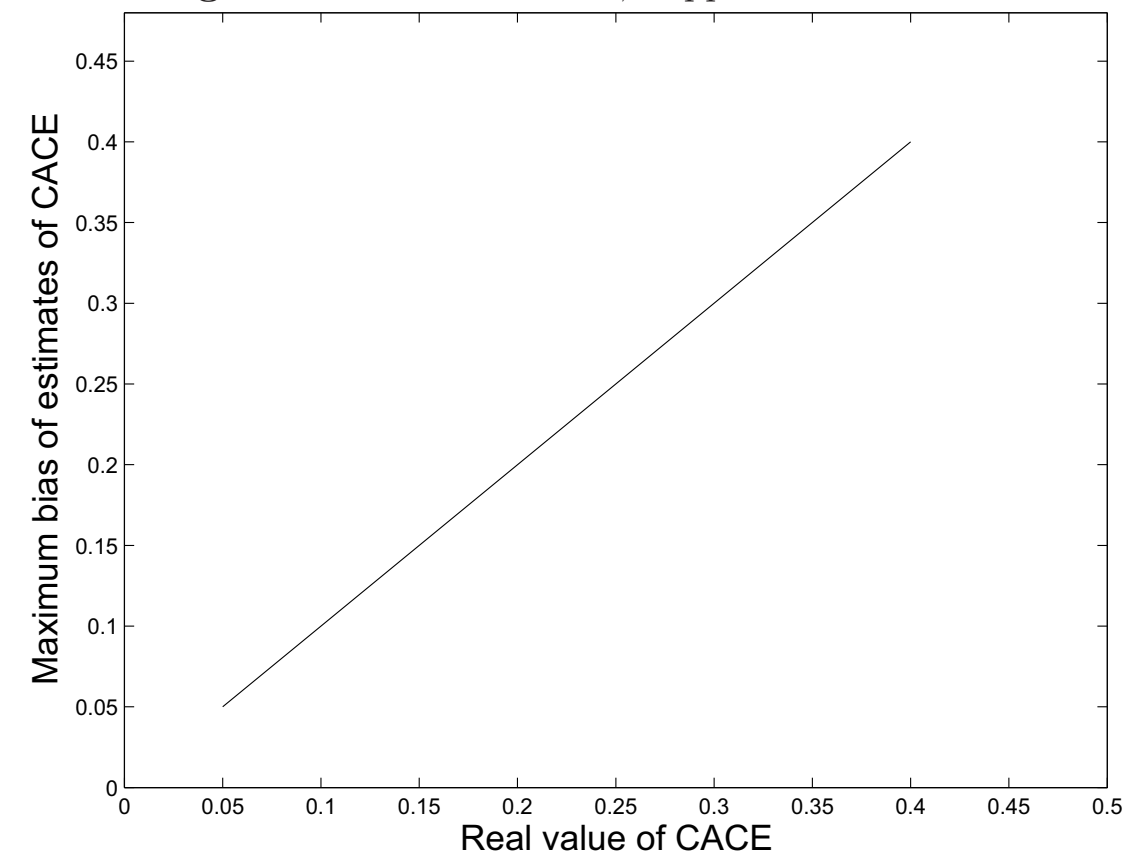


Table 1

Simulation results of comparison between moment method and $M L$ method with covariate in Theorem 2, including mean, standard deviation and actual 95\% coverage probability. The level of covariate is 2 and $N=500$.

\begin{tabular}{lcccccc}
\hline \hline & \multicolumn{3}{c}{ Moment Method } & \multicolumn{3}{c}{ ML Method } \\
Real Parameters & Mean & Std Dev & $95 \%$ Cover & Mean & Std Dev & $95 \%$ Cover \\
\hline$\xi=0.5$ & 0.4998 & 0.0225 & 0.950 & 0.4998 & 0.0225 & 0.950 \\
$P(U=n \mid X=0)=0.3$ & 0.3003 & 0.0451 & 0.949 & 0.2993 & 0.0448 & 0.948 \\
$P(U=a \mid X=0)=0.2$ & 0.2020 & 0.0422 & 0.954 & 0.2008 & 0.0419 & 0.947 \\
$P(U=n \mid X=1)=0.1$ & 0.1007 & 0.0240 & 0.949 & 0.1001 & 0.0239 & 0.950 \\
$P(U=a \mid X=1)=0.5$ & 0.5009 & 0.0397 & 0.949 & 0.4997 & 0.0393 & 0.949 \\
$P(X=0)=0.4$ & 0.3997 & 0.0229 & 0.956 & 0.3997 & 0.0229 & 0.956 \\
$\theta_{10 a 0}=0.6$ & 0.7124 & 0.3501 & 1.000 & 0.6201 & 0.2387 & 0.995 \\
$\theta_{11 n 0}=0.3$ & 0.5013 & 0.3681 & 1.000 & 0.2967 & 0.1169 & 0.961 \\
$\theta_{11 c 0}=0.8$ & 0.6835 & 0.4362 & 1.000 & 0.7673 & 0.1727 & 0.953 \\
$\theta_{10 c 0}=0.2$ & 0.3617 & 0.3806 & 0.832 & 0.2398 & 0.1981 & 0.941 \\
$\theta_{10 a 1}=0.5$ & 0.6372 & 0.3430 & 1.000 & 0.5236 & 0.1690 & 0.966 \\
$\theta_{11 n 1}=0.2$ & 0.4067 & 0.3863 & 0.804 & 0.2014 & 0.1301 & 0.959 \\
$\theta_{11 c 1}=0.7$ & 0.6224 & 0.4610 & 1.000 & 0.6429 & 0.2745 & 0.936 \\
$\theta_{10 c 1}=0.1$ & 0.2005 & 0.2614 & 0.911 & 0.1194 & 0.1052 & 0.956 \\
$\rho_{00}=0.2$ & 0.2331 & 0.2756 & 0.916 & 0.2111 & 0.0507 & 0.950 \\
$\rho_{01}=0.3$ & 0.3357 & 0.2883 & 0.912 & 0.3062 & 0.0793 & 0.952 \\
$\rho_{10}=0.6$ & 0.4103 & 0.2760 & 0.876 & 0.6271 & 0.2145 & 0.999 \\
$\rho_{11}=0.8$ & 0.3515 & 0.3154 & 0.639 & 0.8288 & 0.1237 & 0.997 \\
$C A C E=0.6$ & 0.3823 & 0.6703 & 0.922 & 0.5254 & 0.2928 & 0.933 \\
\hline
\end{tabular}


Table 2

Sensitivity analysis of $C N$ and the method by ignoring incompletely observed subjects. Covariate $X$ is binary and $N=3000$. The true value of $C A C E$ is 0.6.

\begin{tabular}{lllll}
\hline \hline & \multicolumn{2}{c}{$C A C E$} & \multicolumn{2}{c}{$C A C E_{\text {ignor }}$} \\
Value of $\rho_{1}$ & Mean & Std Dev & Mean & Std Dev \\
\hline$\rho_{1}=0.2$ & 0.5923 & 0.0891 & 0.5621 & 0.0867 \\
$\rho_{1}=0.3$ & 0.5992 & 0.0795 & 0.5018 & 0.0715 \\
$\rho_{1}=0.4$ & 0.5977 & 0.0754 & 0.4474 & 0.0611 \\
$\rho_{1}=0.5$ & 0.6001 & 0.0695 & 0.3983 & 0.0547 \\
$\rho_{1}=0.6$ & 0.5987 & 0.0663 & 0.3595 & 0.0490 \\
$\rho_{1}=0.7$ & 0.5947 & 0.0636 & 0.3239 & 0.0455 \\
$\rho_{1}=0.8$ & 0.5965 & 0.0631 & 0.2998 & 0.0410 \\
$\rho_{1}=0.9$ & 0.6017 & 0.0545 & 0.2757 & 0.0376 \\
\hline
\end{tabular}


Table 3

$M L$ estimates and SE of flu shot data.

\begin{tabular}{llcc}
\hline \hline Parameters & MLE & Std Dev(Bootstrap) & $95 \%$ CI \\
\hline$\xi$ & 0.5065 & 0.0097 & $(0.4874,0.5255)$ \\
$\omega_{n}$ & 0.7839 & 0.0108 & $(0.7627,0.8051)$ \\
$\omega_{a}$ & 0.1348 & 0.0091 & $(0.1170,0.1525)$ \\
$\theta_{10 a}$ & 0.1757 & 0.0234 & $(0.1300,0.2215)$ \\
$\theta_{11 n}$ & 0.5216 & 0.0143 & $(0.4936,0.5495)$ \\
$\theta_{11 c}$ & $1.379 \mathrm{e}-016$ & 0.0268 & $(0.0000,0.0526)$ \\
$\theta_{10 c}$ & 0.1393 & 0.1722 & $(0.0000,0.4768)$ \\
$\rho_{0}$ & 1.0000 & 0.0000 & $(1.0000,1.0000)$ \\
$\rho_{1}$ & 0.1151 & 0.0095 & $(0.0965,0.1337)$ \\
$C A C E$ & -0.1393 & 0.1743 & $(-0.4808,0.2022)$ \\
\hline
\end{tabular}

